\title{
Design of Time Aware Traffic Control Cache Algorithm for Efficient Performance of Web Caching
}

\author{
Kavita Kalambe \\ Shri Ramdeobaba College of Engineering \\ and Management, Nagpur, India
}

\begin{abstract}
Web caching plays a vital role to provide better web services and solve the problem of network access delays and network traffic congestion. This paper designed Time Aware Traffic Control Cache replacement algorithm (TATCCA) that saves the network access time by holding those object inside the cache whose access time is more. This considers the access time, object size, frequency, and timespan for choosing the object to be replaced to avoid the cache pollution. Access time is the most prominent factor. The example shows that compared with the traditional cache replacement schemes, the proposed TATCCA strategy has a higher hit rate and provide fastest services to user..
\end{abstract}

KEY WORDS: WWW, WEB CACHING, TATCCA, PROXY SERVER, CACHE MANAGER BLOCK (CMB).

\section{INTRODUCTION}

In recent years, World Wide Web services are highly disseminated among a millions of world population, with heterogeneous interests like marketing, banking, education sector, entertainment, business, personal communication etc. (James Huang and Chao, 2001). Due to this, there has been increase in the network traffic congestion (Kim and Park, 2006) also increase the server load, client latency and bandwidth utilization (Barish and Obraczka, 2000). As every client aspires to get fast network service, the internet must be able to handle an aggressively increasing amount of traffic (N, 2015). Some mechanism should be provide to overcome the network traffic problem. The effective solution is web caching. Web caching is the technique of prefetching and

\section{ARTICLE INFORMATION}

Received 17th Oct 2020 Accepted after revision 29th Dec 2020 Print ISSN: 0974-6455 Online ISSN: 2321-4007 CODEN: BBRCBA

Thomson Reuters ISI Web of Science Clarivate Analytics USA and Crossref Indexed Journal

\section{Clarivate
Analytics}

NAAS Journal Score 2020 (4.31) SJIF: 2020 (7.728)

A Society of Science and Nature Publication,

Bhopal India 2020. All rights reserved.

Online Contents Available at: http//www.bbrc.in/

Doi: http://dx.doi.org/10.21786/bbrc/13.14/22 buffered repository of the web objects closer to clients so that if client request frequently and in the near future is satisfied from the repository located at the client side without interrupting the server (Teng, Chang and Chen, 2005; Ali, Shamsuddin and Ismail, 2011).

The typical structure of the WWW is shown in Fig 1. It includes three elements: cache on the client side, cache on the server side and cache on the proxy server. Proxy server acts as an intermediary between the client and server (Ali, Shamsuddin and Ismail, 2011). Whenever client sends request for the web object, it first arrives at the proxy server instead of the origin server. Proxy server serves these requirements by using either previously stored responses or gain the required document by requesting the original server on behalf of the client. This will reduce network access latency for a web object also reduces network traffic (Arlitt et al., 2000; Barish and Obraczka, 2000).

The overall performance of the system depends on the web cache eviction policies (Bilal and Kang, 2017). Several web cache replacement algorithms have been designed for improving the performance of the system. This paper presents a new approach for Web cache eviction policy by

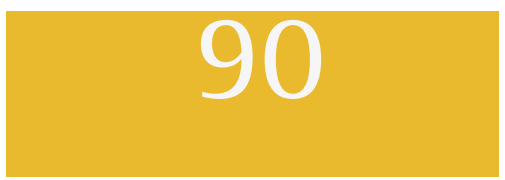


preferentially caching the objects whose cost of retrieval is the largest to improve the network performance as well as provide better service to user.

Figure 1: Architecture of World Wide Web

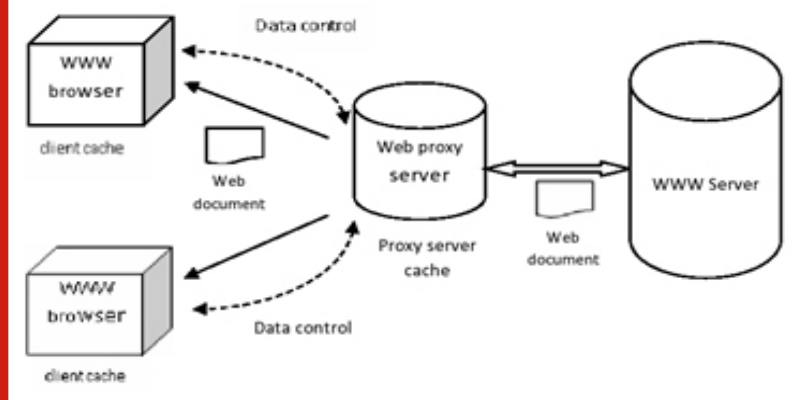

Related Work: Several web cache replacement policies have been proposed for upgrading the network performance. In this, we give an overview of conventional Web Proxy Cache replacement techniques that analyse their performance based on different key parameters used for the eviction and in addition to specify it's limitations.

The Original Greedy Dual Algorithm: The original Greedy dual algorithm developed by Young. In this algorithm, every object of equal size corresponds with cost needed to fetch the objects from secondary storage which is defined by $\mathrm{H}$ value. When cache needs to be replace, then objects with the minimum standard cost Hmin is considered for eviction and decrease the cost values $\mathrm{H}$ of all other objects in the cache by Hmin (Cherkasova, 1998). So object having largest cost value may be next object for the replacement. But it does not consider the non-uniform object size.

1. Greedy Dual Size Algorithm: The Greedy Dual Size algorithm (GDS) (Curcio, Leonardi and Vitaletti, 2002) was introduced by Rizzo and Vicisano. which is the modified version of the Greedy Dual algorithm. This algorithm considers the parameter object size S(i), object cost C(i) and inflation value L( offset value) to calculate the standard key value $\mathrm{H}$ of object(i) and use the priority queue to store $\mathrm{H}(\mathrm{i})$ value. The standard key value for object (i) is calculated in the following way:

$$
\mathrm{H}(\mathrm{i})=\mathrm{L}+\mathrm{C}(\mathrm{i}) / \mathrm{S}(\mathrm{i})
$$

In this algorithm, the object having minimum standard key value Hmin is consider for eviction. The limitation of this algorithm is that it does not consider the frequency value of the object.

2.Greedy Dual Size Frequency Algorithm: To overcome the limitation of GDS algorithm, the Greedy Dual Size Frequency algorithm (GDSF) (Li and Shen, 2004) was proposed that considers the frequency of object $\mathrm{i}$ for compute standard key value of objects in the priority queue. GDSF adds the frequency of object F(i) with the GDS algorithm to calculate the value H(i). The performance of GDSF algorithm is better than GD and GDS algorithm. This algorithm also uses the priority queue. The standard key value for object (i) is calculated in the following way (Li and Shen, 2004):

$$
\mathrm{H}(\mathrm{i})=\mathrm{L}+\mathrm{F}(\mathrm{i}) * \mathrm{C}(\mathrm{i}) / \mathrm{S}(\mathrm{i})
$$

\section{Greedy Dual Size Frequency Access Interest Algorithm:} The GDSF-AI (Sarhan, Elmogy and Ali, 2014) algorithm is considered user's access choice, document types and access trait of the web for efficient web cache replacement policies. In GDSF-AI algorithm, the access choice model for ith web user is represented in the form of vector qi $=\{\langle\mathrm{Ci} 1$, wi1 $>,<\mathrm{Ci} 2$, wi $2>, \ldots,<$ Cin, win $>\}$. Where the set $\{\mathrm{Ci} 1, \mathrm{Ci} 2, . ., \mathrm{Cin}\}$ represents the web user's access choice and the set $\{$ wi1,wi2,..,win $\}$ represents the corresponding weights to the user's access choice. The objects in cache can be specified as the array $\mathrm{Obj}=\{\mathrm{obj} 1, \mathrm{obj} 2, . ., \mathrm{objm}\}$. The interest vector qi of web user $i$ and the vector dj of the jth document, they obtain the two term approximation degree of the documents by using the cosine formula as follows:

$$
\operatorname{SIM}(q i, d j)=\frac{\sum_{k=1}^{n}\left(W d k^{k} W W^{2}\right)}{\sqrt{\sum_{k=1}^{n}\left(W d k^{2}\right)} \cdot \sqrt{\sum_{k=1}^{n}\left(W q k^{2}\right)}}
$$

Proposed Algorithm: While providing fastest CPU request, cache prefetching the requested object from the remote memory. As the cache memory is fastest and limited in capacity, its space must be utilized efficiently so that it will satisfy maximum CPU request. In this paper, we designed a new algorithm called as Time Aware Traffic Control Cache Algorithm (TATCCA). This algorithm selects the object for eviction by considering three parameters i. e. size of object, object fetch time, frequency of object. It maintain a database called Cache Manager Block (CMB) as illustrated with the help of dummy dataset shown in Table 1. Our proposed algorithm computes the Least Effective Object (LE0) (whose size is largest and cost of retrieval is less) large size can be consider either one or summation of size of two object that is largest among the cache database and having less fetch time for eviction based on the Cache Manager Block information.

Table 1. Cache Manager Block (CMB)

\begin{tabular}{|l|c|}
\hline \multicolumn{2}{|c|}{ Object Size(Largest to smallest) } \\
\hline Obj_id & OBJ_Size (in KB) \\
\hline OBJA4 & 32 \\
\hline OBJB2 & 28 \\
\hline OBJB3 & 22 \\
\hline OBJA1 & 16 \\
\hline OBJB1 & 12 \\
\hline OBJA2 & 10 \\
\hline OBJA3 & 8 \\
\hline
\end{tabular}




\section{Descriptions of all the fields of $\mathrm{CMB}$ is as Follows:}

- Obj_id: Obj_id is the object identification number which is used to identify object referred in the cache.

- Size: Size is the size of object.
- Fetch_time: It is the time required to bring the object into the cache from the remote memory.

- Frequency: Frequency of object is the number of times the object is requested by the user.

\begin{tabular}{|c|c|c|c|}
\hline \multicolumn{4}{|c|}{ Table1.2. Database_2(DB_2) } \\
\hline \multicolumn{2}{|c|}{$\begin{array}{l}\text { Fetching Time } \\
\text { (minimum to } \\
\text { maximum) }\end{array}$} & \multirow{2}{*}{$\begin{array}{c}\text { Freque-ncy of } \\
\text { object in cache } \\
\text { Freq }\end{array}$} & \multirow{2}{*}{$\begin{array}{c}\text { Time_Gain } \\
\text { TG=(Fetch_Time* } \\
\text { Freq) -Fetch_Time }\end{array}$} \\
\hline Obj_id & $\begin{array}{l}\text { Fetch_Time } \\
\text { (in ms) }\end{array}$ & & \\
\hline OBJA1 & 25 & 4 & 75 \\
\hline OBJA2 & 41 & 3 & 82 \\
\hline OBJB1 & 52 & 2 & 52 \\
\hline OBJA3 & 71 & 5 & 284 \\
\hline OBJB3 & 95 & 6 & 475 \\
\hline OBJA4 & 101 & 3 & 202 \\
\hline OBJB2 & 122 & 6 & 610 \\
\hline
\end{tabular}

Table1.3. Database_3 (DB_3)

Most recently Evicted Objects and its address in next level memory

\begin{tabular}{|l|c|}
\hline Evicted Obj_id & OBJ_ADDR \\
\hline OBJD1 & 0xED12A367 \\
\hline OBJC2 & 0xABFF1256 \\
\hline OBJB4 & 0x78237698 \\
\hline OBJA5 & 0xDFE98706 \\
\hline OBJC3 & 0x3456AC8B \\
\hline OBJD4 & 0x7865FABC \\
\hline OBJC1 & 0xCCAD7865 \\
\hline
\end{tabular}

\section{Design of TATCCA Algorithm}
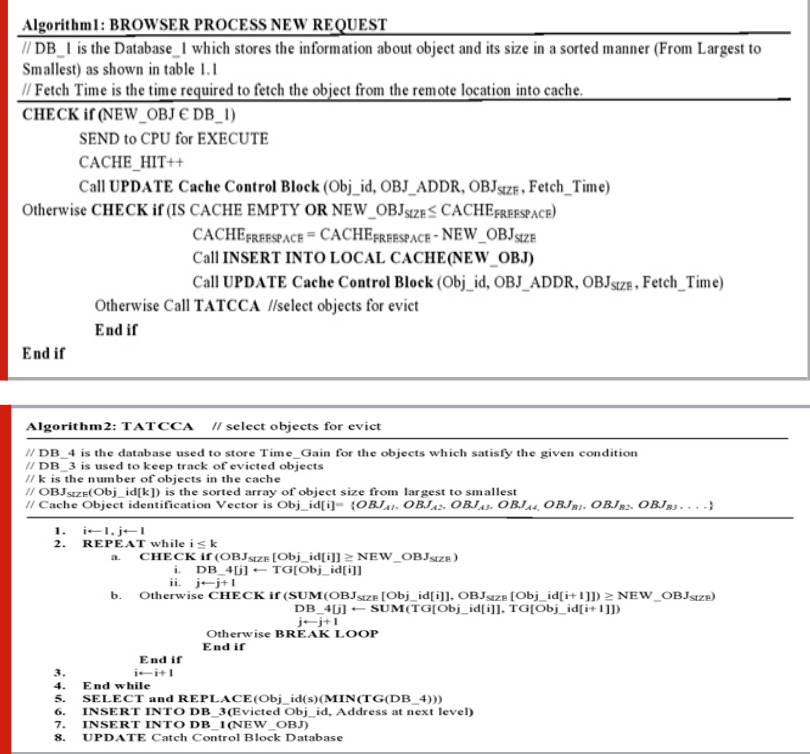

II. lustration of TATCCA Algorithm:Whenever the web user sends the new request onto the network, browser will first search it into DB_1 of CMB. If it is there, then cache hit occurs and it will directly provide to user but if it is not there then cache miss and search it in the DB_3 of CMB to check if it is either evicted recently or not. If requested object is not found in DB_3 also then TATCCA algorithm works when either the Cache is full or have no sufficient space to store the new object. TATCCA works on the basis of information given in the CMB. In Database_2 contains the information about the time required to bring the object from the source into the cache and number of times the object is referred and also the third most important is calculate the time gain factor by using following formula:

Object, TG $($ Obj_id $)=\left[\left(\right.\right.$ Fetch_Time $\left(O b j \_i d\right) \times$ Freq $\left(\operatorname{Obj}_{-}\right.$ id))] - Fetch_Time(Obj_id) ...(1)

This time gain factor helps to choose the least effective object, if the object require maximum time to fetch and if it referred more time then it is very beneficial to keep into the cache for a long time until its comparable object comes into the cache. Our algorithm selects the least effective object whose time gain factor is minimum so that the minimum time spend to fetch object if it is requested again. This will also consider the new object is better to fit into the free space of the cache. This algorithm selects the object in a sequence of largest size to smallest size to see the best fit space for the new object size with reference to select minimum corresponding time gain, this algorithm provides two choices for creating space for new object by selecting either one object having largest size and minimum time_gain or summation of two object's time_gain is minimum and the sum of their size is sufficient to fit the new object. 
Let us consider example if new request arrives OBJC1 which is of size $24 \mathrm{~KB}$, and not found in cache and cache is full, so replacement takes place on the basis of TATCCA algorithm: According to this algorithm and Cache Manager Block information computes least effective object, following Database_4 is created and the object ids OBJA1, OBJB1 is treated as the least effective objects which creates best fit space and their time_gain is also minimum. So if this object is required again then it will take less time to fetch than other objects within the cache.

Table 1.4: Database_4 (DB_4)

\begin{tabular}{|l|c|}
\hline SUM(TG(Obj_id(s))) & Obj_id(s) \\
\hline 202 & OBJA4 \\
\hline 610 & OBJB2 \\
\hline 550 & OBJB3, OBJA1 \\
\hline 127 & OBJA1, OBJB1 \\
\hline
\end{tabular}

Table 1.1: Database_1 (DB_1)

Object Size (Largest to smallest)

\begin{tabular}{|l|c|}
\hline Obj_id & OBJ_Size (in KB) \\
\hline $\mathrm{OBJ}_{\mathrm{A} 4}$ & 32 \\
\hline $\mathrm{OBJ}_{\mathrm{B} 2}$ & 28 \\
\hline $\mathrm{OBJ}_{\mathrm{C} 1}$ & 24 \\
\hline $\mathrm{OBJ}_{\mathrm{B} 3}$ & 22 \\
\hline $0 \mathrm{BJA2}$ & 10 \\
\hline $0 \mathrm{~B} \mathrm{~A}_{\mathrm{A} 3}$ & 8 \\
\hline
\end{tabular}

Table 1.3: Database_3(DB_1)

Most recently Evicted Objects and its address in next level memory

\begin{tabular}{|l|l|}
\hline Evicted Obj_id & OBJ_ADDR \\
\hline $0 B J_{A 1}$ & 0xEDCEA667 \\
\hline $0 \mathrm{BJ}_{\mathrm{B} 1}$ & 0xCBFD125B \\
\hline $0 \mathrm{BJ} \mathrm{D}_{1}$ & 0xED12A367 \\
\hline $\mathrm{OBJ}_{\mathrm{C} 2}$ & 0xABFF1256 \\
\hline $0 \mathrm{BJ} \mathrm{B}_{\mathrm{B} 4}$ & 0x78237698 \\
\hline $\mathrm{OBJ}_{\mathrm{A} 5}$ & 0xDFE98706 \\
\hline $\mathrm{OBJ}_{\mathrm{C} 3}$ & 0x3456AC8B \\
\hline
\end{tabular}

According to the table 1.4 the objects OBJA1, OBJB1 have lowest time gain and summation of its size create best space for the newly requested object OBJC1 having size $24 \mathrm{~KB}$. If largest size object OBJA4 is replaced then it will take 101 time to fetch if it is requested again. Though OBJA4 has less frequency than OBJA1 still it is better to replace OBJA1, OBJB1 who require only 25 and 52 time to fetch respectively. Due to this process we will save time to access object from remote memory and one or more object can be replaced to create large space in the cache. After replacement new status of CMB database_1 is shown in table 1.1 and reference of replaced object is also save in database_3 shown in table 1.3. This algorithm will save reference of recently replaced object so that after some time if OBJA1 or OBJB1 is requested again then it will be fetched from referenced location directly so that this will save searching time and accessing quickly.

\section{CONCLUSION}

Web caching is one of the most promising techniques to reduce network access delay, network traffic congestion, bandwidth utilization. Nonetheless, most of the related work in the literature is about improvements of Web cache replacement algorithm, when the Internet was very different from todays. Starting from this observation, this paper aim to provide new viewpoints on the web cache replacement algorithm i. e. designed Time Aware Traffic Control Cache algorithm (TATCCA) in order to make caching i) efficient in enhancing the metrics that are the most relevant for the fastest network, in particular satisfying users demands quickly, ii) suitable for the type of different file that largely dominates the traffic today, e.g. video, audio and iii) provide quick response to user demands and save access time. In this algorithm, the objects whose has highest frequency and access time, it is affordable to keep those objects inside the cache so that whenever it is requested again then due to this algorithm cache hit occur and save its access time also. This algorithm is better to provide fastest network services.

\section{REFERENCES}

Ali, W., Shamsuddin, S. M. and Ismail, A. S. (2011) 'A survey of web caching and prefetching', International Journal of Advances in Soft Computing and its Applications, 3(1), pp. 18-44.

Arlitt, M. et al. (2000) 'Evaluating content management techniques for web proxy caches', Performance Evaluation Review, 27(4), pp. 3-11. doi: 10.1145/346000.346003. Barish, G. and Obraczka, K. (2000) 'World wide web caching: Trends and techniques', IEEE Communications Magazine, 38(5), pp. 178-184. doi: 10.1109/35.841844.

Beckmann, N., Chen, H. and Cidon, A. (2018) 'LHD: Improving cache hit rate by maximizing hit density', in Proceedings of the 15th USENIX Symposium on Networked Systems Design and Implementation, NSDI 2018.

Bilal, M. and Kang, S. G. (2017) 'A Cache Management Scheme for Efficient Content Eviction and Replication in Cache Networks', IEEE Access. doi: 10.1109/ ACCESS.2017.2669344.

Chao, W. (2020) 'Web cache intelligent replacement 
strategy combined with GDSF and SVM network reaccessed probability prediction', Journal of Ambient Intelligence and Humanized Computing. Springer Berlin Heidelberg, 11(2), pp. 581-587. doi: 10.1007/s12652018-1109-4.

Cherkasova, L. (1998) 'Improving WWW proxies performance with Greedy-Dual-Size-Frequency caching policy', HP Laboratories Technical Report, (98-69).

Cherkasova, L. and Ciardo, G. (2001) 'Role of aging, frequency, and size in web cache replacement policies', in Lecture Notes in Computer Science (including subseries Lecture Notes in Artificial Intelligence and Lecture Notes in Bioinformatics). doi: 10.1007/3-54048228-8_12.

Curcio, M., Leonardi, S. and Vitaletti, A. (2002) 'An experimental study of prefetching and caching algorithms for the world wide web', Lecture Notes in Computer Science (including subseries Lecture Notes in Artificial Intelligence and Lecture Notes in Bioinformatics), 2409, pp. 71-85. doi: 10.1007/3-54045643-0_6.

Du, J. et al. (2018) 'A Web cache replacement strategy for spacecraft comprehensive testing system', Beijing Hangkong Hangtian Daxue Xuebao/Journal of Beijing University of Aeronautics and Astronautics, 44(8), pp. 1609-1619. doi: 10.13700/j.bh.1001-5965.2017.0591.

James Huang, C. and Chao, M. H. (2001) 'Managing WWW in public administration: Uses and misuses', Government Information Quarterly. doi: 10.1016/S0740624X(01)00085-5.

Jeong, J. and Dubois, M. (2006) 'Cache replacement algorithms with nonuniform miss costs', IEEE Transactions on Computers. doi: 10.1109/TC.2006.50. Kim, K. and Park, D. (2006) 'Reducing outgoing traffic of proxy cache by using client-cluster', Journal of Communications and Networks, 8(3), pp. 330-338. doi: 10.1109/JCN.2006.6182773.

Li, K. and Shen, H. (2004) 'An improved Greedy Dual* cache document replacement algorithm', in Proceedings - IEEE/WIC/ACM International Conference on Web Intelligence, WI 2004.

Lipasti, P. M. H. (2016) 'Cache Replacement Policies Cache Design : Four Key Issues'.

Ma, T. et al. (2018) 'Weighted greedy dual size frequency based caching replacement algorithm', IEEE Access. doi: 10.1109/ACCESS.2018.2790381.

N, É. D. (2015) 'These De Doctorat De L' U Niversite P Aris -S Aclay, Preparee a L' U Niversite P Aris -S Ud'.

Paschos, G. S. et al. (2018) 'The role of caching in future communication systems and networks', IEEE Journal on Selected Areas in Communications, 36(6), pp. 1111-1125. doi: 10.1109/JSAC.2018.2844939.

Rizzo, L. and Vicisano, L. (2000) 'Replacement policies for a proxy cache', IEEE/ACM Transactions on Networking. doi: 10.1109/90.842139.

Sarhan, A., Elmogy, A. M. and Ali, S. M. (2014) 'New Web cache replacement approaches based on internal requests factor', Proceedings of 2014 9th IEEE International Conference on Computer Engineering and Systems, ICCES 2014, 15(3), pp. 383-389. doi: 10.1109/ ICCES.2014.7030990.

Singh, D. D., Kumar, S. and Kapoor, S. (2011) 'An Explore View of Web Caching Techniques', International Journal of Advances in Engineering Sciences, 1(3), pp. 38-43. Available at: http://citeseerx.ist.psu.edu/viewdoc/summ ary?doi=10.1.1.682.6996\%0Ahttp://www.rgsociety.org/ journals/index.php/ijse/article/view/175.

Teng, W. G., Chang, C. Y. and Chen, M. S. (2005) 'Integrating web caching and web prefetching in client-side proxies', IEEE Transactions on Parallel and Distributed Systems, 16(5), pp. 444-455. doi: 10.1109/ TPDS.2005.56.

Wong, K. Y. (2006) 'Web cache replacement policies: A pragmatic approach', IEEE Network, 20(1), pp. 28-34. doi: 10.1109/MNET.2006.1580916.

$\mathrm{Xu}$, J. et al. (2004) 'Caching and prefetching for web content distribution', Computing in Science and Engineering, 6(4), pp. 54-59. doi: 10.1109/ MCSE.2004.5.

Yang, Q. et al. (2001) 'Mining web logs to improve web caching and prefetching', in Lecture Notes in Computer Science (including subseries Lecture Notes in Artificial Intelligence and Lecture Notes in Bioinformatics). doi: 10.1007/3-540-45490-x_62.

Zhao, Z., Ma, Y. and Cong, Q. (2018) 'GDSF-based low access latency web proxy caching replacement algorithm', in ACM International Conference Proceeding Series. doi: 10.1145/3297156.3297237. 